International Journal of Pure and Applied Mathematics

Volume 108 No. 4 2016, 859-864

ISSN: 1311-8080 (printed version); ISSN: 1314-3395 (on-line version)

url: http://www.ijpam.eu

doi: 10.12732/ijpam.v108i4.11

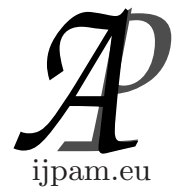

\title{
SOME RESULTS OF DUALITY IN \\ FUZZY LINEAR PROGRAMMING
}

\author{
S. Hosaeni ${ }^{1}$, J. Cheshmavar ${ }^{2} \S$ \\ ${ }^{1,2}$ Department of Mathematics \\ College of Sciences \\ Payame Noor University \\ P.O. Box 19395-3697, Tehran, IRAN
}

\begin{abstract}
In this paper, based on linear ranking functions we investigate some properties for the fuzzy number linear programming problems and its dual with the relationships between them.
\end{abstract}

AMS Subject Classification: 90C05, 90C46, 90C70

Key Words: fuzzy numbersm fuzzy linear programming, ranking functions, duality

\section{Introduction}

In the real world decision problems with the linear programming models and the crisp parameters may be exists some uncertainty, a decision maker does not always know the exact values of the parameters taking part in the problem, and that vagueness in the parameters may not be of a probabilistic type. In this situation, the decision maker can model the inexactness by means of fuzzy parameters. Of course a number of researchers have exhibited interest in the topic fuzzy linear programming. this concept was first proposed by Bellman and Zadeh [2]. Maleki et al. [5, 6] investigated the construction of linear

Received: October 18, 2015

Published: August 16, 2016

${ }^{\S}$ Correspondence author (c) 2016 Academic Publications, Ltd.

url: www.acadpubl.eu 
programming with fuzzy variables, and solving fuzzy linear programming with certain ranking function, On duality in linear programming under fuzzy environment [1], and Mishmast et al. the lexicographic ranking function to order fuzzy numbers and solved them [7]. Here we investigate some properties for the fuzzy number linear programming problems and its dual.

\section{Some Notation and Preliminaries}

Throughout this paper, we use fundamental notations initiated by Bellman and Zadeh [2], and also can be found in [4].

Definition 2.1. Let $X$ is a collection of objects denoted generally by $x$, then a fuzzy set $\widetilde{A}$ in $X$ is defined to be a set of ordered pairs $\widetilde{A}=\left\{\left(x, \mu_{\widetilde{A}}(x)\right)\right.$ : $x \in X\}$, where $\mu_{\widetilde{A}}(x)$ is called the membership function of $x$ in $\widetilde{A}$. The membership function maps each elements of $X$ to a membership value between 0 and 1.

Definition 2.2. The $\alpha$-level set of $\widetilde{A}$ is the set $\widetilde{A}_{\alpha}=\left\{x \in \mathbb{R}: \mu_{\widetilde{A}}(x) \geq \alpha\right\}$, where $\alpha \in(0,1]$.

Definition 2.3. A fuzzy set $\widetilde{A}$ is convex if

$$
\mu_{\widetilde{A}}(\lambda x+(1-\lambda) y) \geq \min \left\{\mu_{\widetilde{A}}(x), \mu_{\widetilde{A}}(y)\right\}, \quad \forall x, y \in X, \text { and } \lambda \in[0,1] .
$$

Definition 2.4. A convex fuzzy set $\widetilde{A}$ on $\mathbb{R}$ is a fuzzy number if the following conditions hold: (i) Its membership function is piecewise continuous. (ii) There exists only $x_{0}$ that $\mu_{\widetilde{A}}\left(x_{0}\right)=1$.

\section{Ranking Functions and Some Relations between $F N L P$ and $D F N L P$}

The ranking function is denoted by $\Re$ where $\Re: F(\Re) \rightarrow \mathbb{R}$ and $F(\Re)$ is the set of fuzzy numbers defined on the real line. It's a method for comparison of fuzzy numbers, of course several methods for solving FNLP can be seen in Fang [3], Maleki et al. [6], and Tanaka [8], one of the most convenient introduced by Maleki [5]. Suppose that $\tilde{a}$ and $\tilde{b}$ be two fuzzy numbers, then the ranking function of $F(\Re)$ is as follows:

$$
\tilde{a} \geq_{\Re} \tilde{b} \text { if and only if } \Re(\tilde{a}) \geq \Re(\tilde{a}),
$$




$$
\begin{aligned}
& \tilde{a}>_{\Re} \tilde{b} \text { if and only if } \Re(\tilde{a})>\Re(\tilde{a}), \\
& \tilde{a}=_{\Re} \tilde{b} \text { if and only if } \Re(\tilde{a})=\Re(\tilde{a}) .
\end{aligned}
$$

where $\tilde{a}$ and $\tilde{b}$ are in $F(\Re)$.

Definition 3.1. A fuzzy number linear programming problem (FNLP) is defined as follows:

$$
\begin{gathered}
\max \tilde{z}=\Re \sum_{j=1}^{n} \tilde{c_{j}} x_{j} \\
\text { s.t. } \quad \sum_{j=1}^{n} \tilde{a}_{i j} x_{j} \quad \leq_{\Re} \tilde{b}_{i}, \quad i=1,2, \cdots m, \\
x_{j} \geq 0, \quad j=1,2, \cdots n .
\end{gathered}
$$

The dual fuzzy number linear programming problem ( $D F N L P$ ) define as

$$
\begin{aligned}
\min \tilde{y}=\Re \sum_{i=1}^{m} \omega_{i} \tilde{b}_{i} \\
\text { s.t. } \quad \sum_{i=1}^{m} \omega_{i} \tilde{a}_{i j} \geq_{\Re} \tilde{c}_{j}, \quad j=1,2, \cdots n, \\
\omega_{i} \geq 0, \quad i=1,2, \cdots m .
\end{aligned}
$$

where $=\Re$ and $\leq_{\Re}$ mean equality and inequality with respect to the ranking function $\Re, \widetilde{A}=\left(\tilde{a}_{i j}\right)_{m \times n}, \tilde{b}=\left(\tilde{b_{1}}, \tilde{b_{2}}, \cdots \tilde{b_{m}}\right), \tilde{c}=\left(\tilde{c_{1}}, \tilde{c_{2}}, \cdots \tilde{c_{n}}\right)$, and $\tilde{a}_{i j}, \tilde{b_{i}}, \tilde{c_{j}} \in$ $F(\Re)$ for $i=1, \cdots, m, j=1, \cdots, n$.

Not that, any $x$ and $\omega$ which satisfies the set of constraints of FNLP and $D F N L P$ is called a feasible solutions respectively. Let $\Omega$ and $\Gamma$ be the set of all feasible solutions of $F N L P$ and $D F N L P$, respectively, then weak and strong duality holds for DFNLP as follows, (see [4]).

Theorem 3.2. (Weak duality) If $x_{0}$ and $\omega_{0}$ are feasible solutions to FNLP and DFNLP, respectively, then $\tilde{c} x_{0} \leq_{\Re} \omega_{0} \tilde{b}$.

Theorem 3.3. (Strong duality) If any one of the FNLP or DFNLP has an optimal solution, then both problems have optimal solutions and the two optimal values of ranking functions for the fuzzy objectives are equal.

Applying the above theorem, we have the following an important result of duality, 
Theorem 3.4. Assume that the optimal objective value of FNLP is equal to the optimal objective value of DFNLP, then $x^{*} \in \Omega$ solves FNLP and $\omega^{*} \in \Gamma$ solves DFNLP if and only if

$$
\sum_{j=1}^{n} \tilde{a}_{i j} x_{j}^{*}-\tilde{b}_{i}=\Re 0, \quad i=1,2, \cdots, m,
$$

and

$$
\tilde{c_{j}}-\sum_{i=1}^{m} \tilde{a}_{i j} \omega_{i}^{*}=\Re 0, j=1,2, \cdots, n .
$$

Proof. Let $x^{*} \in \Omega$ and $\omega^{*} \in \Gamma$. Then we have

$$
\tilde{c_{j}} \leq_{\Re} \sum_{i=1}^{m} \tilde{a}_{i j} \omega_{i}^{*}, j=1,2, \cdots, n .
$$

and

$$
\sum_{j=1}^{n} \tilde{a}_{i j} x_{j}^{*} \leq_{\Re} \tilde{b}_{i}, \quad i=1,2, \cdots, m,
$$

or

$$
\Re\left(\tilde{c}_{j}\right) \leq \sum_{i=1}^{m} \Re\left(\tilde{a}_{i j}\right) \omega_{i}^{*}, \quad j=1,2, \cdots, n .
$$

and

$$
\sum_{j=1}^{n} \Re\left(\tilde{a}_{i j}\right) x_{j}^{*} \leq \Re\left(\tilde{b}_{i}\right), \quad i=1,2, \cdots, m,
$$

Thus,

$$
\begin{gathered}
\sum_{j=1}^{n} \Re\left(\tilde{c}_{j}\right) x_{j}^{*} \leq \sum_{j=1}^{n}\left(\sum_{i=1}^{m} \Re\left(\tilde{a}_{i j}\right) \omega_{i}^{*}\right) x_{j}^{*}= \\
\sum_{i=1}^{m}\left(\sum_{j=1}^{n} \Re\left(\tilde{a}_{i j}\right) x_{j}^{*}\right) \omega_{i}^{*} \leq \sum_{i=1}^{m} \omega_{i}^{*} \Re\left(\tilde{b}_{i}\right)
\end{gathered}
$$

Now if $x^{*}$ and $\omega^{*}$ are optimal solutions to $F N L P$ and $D F N L P$, respectively, then by the assumption that the optimal objective value of $F N L P$ is equal to the optimal objective value of $D F N L P$, we have 


$$
\sum_{j=1}^{n} \tilde{c_{j}} x_{j}^{*}=\Re \sum_{i=1}^{m} \omega_{i}^{*} \tilde{b_{i}}
$$

or

$$
\sum_{j=1}^{n} \Re\left(\tilde{c}_{j}\right) x_{j}^{*}=\sum_{i=1}^{m} \omega_{i}^{*} \Re\left(\tilde{b}_{i}\right),
$$

Therefor, by 3.3, the identity 3.5 and 3.6 holds if

$$
\sum_{j=1}^{n} \Re\left(\tilde{a}_{i j}\right) x_{j}^{*}-\Re\left(\tilde{b}_{i}\right)=0
$$

and

$$
\Re\left(\tilde{c}_{j}\right)-\sum_{i=1}^{m} \Re\left(\tilde{a}_{i j}\right) \omega_{i}^{*}=0,
$$

or

$$
\sum_{j=1}^{n} \tilde{a}_{i j} x_{j}^{*}-\tilde{b}_{i}=\Re 0
$$

and

$$
\tilde{c_{j}}-\sum_{i=1}^{m} \tilde{a}_{i j} \omega_{i}^{*}=\Re 0 .
$$

This proves the theorem and the converse of the theorem follows similarly.

\section{Acknowledgments}

We are thankful to the anonymous referees.

\section{References}

[1] C.R. Bector, S. Chadra, On duality in linear programming under fuzzy environment, Fuzzy Sets Sys., 125 (2002), 317-325. 
[2] R.E. Bellman, L.A. Zadeh, Desion making in a fuzzy environment, Manag. Sci., 1 (1993), $1-9$.

[3] S.C. Fang, C.F. Hu, Linear programming with fuzzy coeffcients in constraint, Comp. math. Appl., 37 (1999), 63-76.

[4] N. Mahdavi-Amiri, S.H. Nasseri, Duality in fuzzy linear programing by use of a certain linear ranking function, Appl. Math. Comp., 180 (2006), 206-216.

[5] H.R. Maleki, M. Tata, M. Mashinchi, Linear programming with fuzzy variables, Fuzzy Sets Sys., 109 (2000), 21-33.

[6] H.R. Maleki, ranking functions and their applications to fuzzy linear programming, Far East J. Math. Sci., 4 (2002), 283-301.

[7] H. Mishmast, H.R. Maleki, M. Mashinchi, Solving fuzzy number linear programming by lexico-graphic ranking function, Italian J. Pure Appli. Math., 15 (2004), 9-20.

[8] H. Tanaka, H. Ichihashi, A formulation of fuzzy linear programming problem based on comparison of fuzzy numbers, Control Cyber., 13 (1984), 185-194. 